\title{
A note on standard composition algebras of types II and III
}

\author{
Beites, P. D. and Nicolás’ A. P.
}

March 28, 2016

\begin{abstract}
Some identities satisfied by certain standard composition algebras, of types II and III, are studied and become candidates for the characterization of the mentioned types. Composition algebras of arbitrary dimension, over a field $F$ with $\operatorname{char}(F) \neq 2$ and satisfying the identity $x^{2} y=n(x) y$ are shown to be standard composition algebras of type II. As a consequence, the identity $y x^{2}=n(x) y$ characterizes the type III.
\end{abstract}

Keywords: standard composition algebra, identity

2010 Mathematics Subject Classification: 17A75, 15A21

\section{Introduction}

Among composition algebras, the well known ones are those with identity, that is, Hurwitz algebras. These over a field of characteristic different from 2, by the generalized Hurwitz Theorem in [5], are isomorphic either to the base field, a separable quadratic extension of the base field, a generalized quaternion algebra or a generalized Cayley algebra. In particular, the dimension of any Hurwitz algebra is 1,2,4 or 8 . This restriction on the dimension was also proved by Chevalley using Clifford algebras (for more details, see [3]).

In general, the classification of all finite dimensional composition algebras is still an open problem. Nevertheless, the imposition of one or more additional conditions on these algebras, in the form of identities, can afford such a classification. For instance, in [8], Okubo proved that, over fields of characteristic different from 2 , any finite dimensional composition algebra

*corresponding author, Centro de Matemática e Aplicações and Departamento de Matemática, Universidade da Beira Interior, Portugal

${ }^{\dagger}$ Instituto de Investigación en Matemáticas and Departamento de Matemática Aplicada, Universidad de Valladolid, Spain 
satisfying the flexible identity $(x y) x=x(y x)$ is either a form of a Hurwitz algebra, a form of a para-Hurwitz algebra or an Okubo algebra.

In [1] we began to approach the problem of characterizing standard composition algebras of type II over a field $F$ with $\operatorname{char}(F) \neq 2$. Concretely, we applied the classification of 2-dimensional and of 4-dimensional composition algebras due to, respectively, Petersson, in [9], and Stampfli-Rollier, in [10]. Unfortunately, since no similar description is known for 8-dimensional composition algebras, the strategy in [1] could not be continued.

In the present work we consider the standard composition algebras of types II and III, over a field $F$ with $\operatorname{char}(F) \neq 2$, associated to the Hurwitz algebras $\mathbb{H}$ (quaternion algebra) and $\mathbb{D}$ (octonion algebra). Both $\mathbb{H}$ and $\mathbb{O}$ are related to certain Clifford algebras, as can be read, for instance, in [7] and references therein. In Sections 3 and 4, we focus on some identities satisfied by the former algebras and, concretely for the ones of degree 3, we apply the random vectors method. See [2] for more details about the cited process that involves computational linear algebra on matrices.

In Section 5, we analyze if the obtained identities characterize standard composition algebras of types II and III. On the one hand, we see that the degree 3 identity that implies all degree 3 identities of the considered standard composition algebras of type II, associated either to $\mathbb{H}$ or to $\mathbb{O}$, can not accomplish that purpose for the mentioned type. The same happens with the corresponding one for type III. On the other hand, we conclude, in arbitrary dimension and over a field of characteristic different from 2 , that the identity $x^{2} y=n(x) y$ (respectively, $y x^{2}=n(x) y$ ) characterizes the standard composition algebras of type II (respectively, III).

\section{Preliminaries}

From now on, with exception of Theorem 3.2 and Theorem 4.2, $F$ is a field such that $\operatorname{char}(F) \neq 2$.

Let $A$ be an algebra over $F$, with multiplication denoted by juxtaposition.

An identity of $A$ has degree $s$ (i.e., is a $s$-identity), where $s \in \mathbb{N}$ and $s \geq 2$, if the multiplication appears $s-1$ times in each term of the identity.

The opposite algebra $A^{o p}$ of $A$ is the algebra with the same underlying vector space as $A$ but with multiplication defined by $x \circ y=y x$.

The algebra $A$ is a composition algebra if it is endowed with a nondegenerate quadratic form (the norm) $n: A \rightarrow F$ which is multiplicative, i.e., for any $x, y \in A$,

$$
n(x y)=n(x) n(y) .
$$


The form $n$ being nondegenerate means that the associated symmetric bilinear form $n(x, y)=\frac{1}{2}(n(x+y)-n(x)-n(y))$ is nondegenerate.

Recall that from (1), by linearization, for all $x, y, z \in A$,

$$
n(x y, x z)=n(x) n(y, z)=n(y x, z x)
$$

that is, the linear maps of left and right multiplication by $x, L_{x}: y \mapsto x y$ and $R_{x}: y \mapsto y x$, are similarities of the norm $n(x)$. As a consequence, $L_{x}$ and $R_{x}$ are injective for all $x \in A$ such that $n(x) \neq 0$.

A unital composition algebra, that is, a composition algebra with identity is called a Hurwitz algebra.

Hurwitz algebras are the main ingredient in the construction of finite dimensional composition algebras without identity. In fact, given a Hurwitz algebra with multiplication $*$, norm $n$, and $\phi, \psi$ two isometries of $n$, the multiplication

$$
x y=\phi(x) * \psi(y)
$$

defines a new composition algebra with the same norm $n$ but, generally, nonunital, [6]. Conversely, given a finite dimensional composition algebra $A$ with product denoted by juxtaposition and quadratic form $n$, for any element $a$ with $n(a) \neq 0$, the left and the right multiplication operators $L_{u}$ and $R_{u}$, where $u=\frac{a^{2}}{n(a)}$, are isometries of $n$. Hence, the new multiplication

$$
x * y=\left(R_{u}^{-1}(x)\right)\left(L_{u}^{-1}(y)\right)
$$

gives a Hurwitz algebra with norm $n$ and identity $u^{2},[6]$. So, the dimension of any finite dimensional composition algebra is equal to $1,2,4$ or 8 .

According to [4], modifying the multiplication $*$ of a Hurwitz algebra $(A, *)$ over an algebraically closed field as in (2) leads to a new composition algebra, relative to the same quadratic form $n$, with "fewer degrees of symmetry" except for the multiplications defined by

$$
\begin{array}{llll}
\text { (I) } x * y & \text { (II) } \bar{x} * y & \text { (III) } x * \bar{y} & \text { (IV) } \bar{x} * \bar{y},
\end{array}
$$

where $x \mapsto \bar{x}=n(x, 1) 1-x$ denotes the standard involution of $(A, *)$ with $n(x) 1=x * \bar{x}=\bar{x} * x$ and $x+\bar{x}=T(x) 1$. The new algebras are called standard composition algebras of the corresponding type, that is, I, II, III, $\mathrm{IV}$, respectively, associated to $(A, *)$. If the dimension of $A$ is 1 , then all standard composition algebras are the base field $F$. In higher dimensions, standard composition algebras of different type are not isomorphic. 
Kaplansky, in [6], proved that every Hurwitz algebra must be finite dimensional. However, there exist infinite dimensional composition algebras, necessarily non-unital. Namely, as mentioned in [4], Elduque and PérezIzquierdo gave examples of such algebras with a one-sided identity element.

\section{Some standard composition algebras asso- ciated to the quaternion algebra $(\mathbb{H}, *)$}

From now on, we can suppose, extending scalars if necessary, that $F$ is algebraically closed. Let $(\mathbb{H}, *)$ be the quaternion algebra over $F$ with basis $\left\{e_{0}, e_{1}, e_{2}, e_{3}\right\}$ and multiplication table given by $e_{1} * e_{1}=e_{2} * e_{2}=e_{3} * e_{3}=$ $e_{1} * e_{2} * e_{3}=-e_{0}$, where $e_{0}$ is the identity. We now consider $\mathcal{H}$, the standard composition algebra of type II associated to $(\mathbb{H}, *)$, over $F$. Its multiplication, denoted by juxtaposition, is given by

$$
x y:=\bar{x} * y .
$$

Theorem 3.1. Let $\mathcal{H}$ be the standard composition algebra of type II associated to $(\mathbb{H}, *)$ over an algebraically closed field $F$ with $\operatorname{char}(F) \neq 2$. There is no 2 -identity satisfied by $\mathcal{H}$.

Proof. The 2-identities satisfied by $\mathcal{H}$ are of the form $\zeta x y+\eta y x=0$, where $\zeta$ and $\eta$ are scalars. With $x=e_{0}$, we obtain $\zeta y+\eta \bar{y}=0$ for all $y$. If any of the scalars is non-null, then $y$ and $\bar{y}$ are linearly dependent, which is a contradiction. Hence $\zeta=\eta=0$.

Consider the following table.

\begin{tabular}{|r|r|r|r|r|}
\hline & $e_{0}$ & $e_{1}$ & $e_{2}$ & $e_{3}$ \\
\hline$e_{0}$ & $e_{0}$ & $e_{1}$ & $e_{2}$ & $e_{3}$ \\
\hline$e_{1}$ & $-e_{1}$ & $e_{0}$ & $-e_{3}$ & $e_{2}$ \\
\hline$e_{2}$ & $-e_{2}$ & $e_{3}$ & $e_{0}$ & $-e_{1}$ \\
\hline$e_{3}$ & $-e_{3}$ & $-e_{2}$ & $e_{1}$ & $e_{0}$ \\
\hline
\end{tabular}

Table 1: Multiplication table of $\mathcal{H}$, where $e_{0}$ is its left identity.

Let $a=\sum_{i=0}^{3} a_{i} e_{i} \in \mathcal{H}$. Let $l(a)$ and $r(a)$ denote, respectively, the coordinate matrices of the left multiplication by $a$ and of the right multiplication by $a$. Then, calculating $a x$ and $x a$, we have 


$$
l(a)=\left[\begin{array}{rrrr}
a_{0} & a_{1} & a_{2} & a_{3} \\
-a_{1} & a_{0} & a_{3} & -a_{2} \\
-a_{2} & -a_{3} & a_{0} & a_{1} \\
-a_{3} & a_{2} & -a_{1} & a_{0}
\end{array}\right] \text { and } r(a)=\left[\begin{array}{rrrr}
a_{0} & a_{1} & a_{2} & a_{3} \\
a_{1} & -a_{0} & -a_{3} & a_{2} \\
a_{2} & a_{3} & -a_{0} & -a_{1} \\
a_{3} & -a_{2} & a_{1} & -a_{0}
\end{array}\right]
$$

Theorem 3.2. Let $\mathcal{H}$ be the standard composition algebra of type II associated to $(\mathbb{H}, *)$ over an algebraically closed field $F$ with $\operatorname{char}(F)=0$. The 3-identities satisfied by $\mathcal{H}$ are consequences of

$$
v^{2} u=(u, v, v)+v(u v),
$$

where $(\cdot, \cdot, \cdot)$ denotes the associator in $\mathcal{H}$.

Proof. In degree 3 , there are two association types, $(\cdot) \cdot$ and $\cdot(\cdot)$, i.e., the 3 -identities of $\mathcal{H}$ are of the form

$$
\sum_{\sigma \in \mathcal{S}_{3}}\left(\alpha_{\sigma}\left(x_{\sigma(1)} x_{\sigma(2)}\right) x_{\sigma(3)}+\beta_{\sigma} x_{\sigma(1)}\left(x_{\sigma(2)} x_{\sigma(3)}\right)\right)=0,
$$

where $\alpha_{\sigma}, \beta_{\sigma} \in F$ and $\mathcal{S}_{3}$ stands for the symmetric group of degree 3 . We now apply the random vectors method, using Maple ${ }^{\mathrm{TM}}$, in characteristic 0 . From the previous notations, we use $l(x) Y$ for the multiplication given by $x y$, where $Y=\left[y_{0} \ldots y_{3}\right]^{T}$.

The process begins with the construction of a $16 \times 12$ matrix $M$ initialized to zero. The first 6 columns and columns 7 to 12 of $M$ are labeled by the 6 monomials $(\cdot \cdot) \cdot$ and by the 6 monomials $\cdot(\cdot \cdot)$, respectively, in (4). Furthermore, we think of $M$ as consisting of a $12 \times 12$ square matrix on top of a $4 \times 12$ matrix. We generate three pseudo-random vectors with 4 components. We allocate the 4 components of the evaluation of the $j$ th $(j \in\{1, \ldots, 6\})$ monomial $(\cdot \cdot) \cdot$ and of the $j$ th $(j \in\{7, \ldots, 12\})$ monomial - (. .) in column $j$ of $M$, in rows 13 to 16 . The computation of the row canonical form of the obtained matrix completes the first iteration of the algorithm. We repeat this fill and reduce process until the stabilization of the rank of the matrix is reached, arriving at rank 10. The 3-identities satisfied by $\mathcal{H}$ lie in the 2 -dimensional nullspace of this matrix. Expressing the vectors of a basis for this subspace as linear combinations of the original 12 monomials, we obtain the subsequent identities

$$
\begin{aligned}
& -(x z) y+(y x) z+(y z) x-(z x) y+x(y z)-y(x z)-y(z x)+z(y x)=0, \\
& -(y z) x+(x y) z+(x z) y-(z y) x+y(x z)-x(y z)-x(z y)+z(x y)=0 .
\end{aligned}
$$

It is clear that the second identity can be obtained by the action of the transposition $(x y)$ over the first one. So, the first identity generates the whole space of 3 -identities of $\mathcal{H}$ under the action of $\mathcal{S}_{3}$. 
Linearizing (3), we see that all 3-identities satisfied by $\mathcal{H}$ are implied by (3).

Although $x^{2} y=n(x) y$ is not an identity of a certain degree $s$, in the sense defined in the Preliminaries, this condition is satisfied by $\mathcal{H}$.

Proposition 3.3. Let $\mathcal{H}$ be the standard composition algebra of type II associated to $(\mathbb{H}, *)$ over an algebraically closed field $F$ with $\operatorname{char}(F) \neq 2$. The condition $x^{2} y=n(x) y$ is an identity of $\mathcal{H}$.

Proof. Notice that $x^{2} y=\overline{(\bar{x} * x)} * y=n(x) y$.

Theorem 3.4. Let $\mathcal{H}$ be the standard composition algebra of type II associated to $(\mathbb{H}, *)$ over an algebraically closed field $F$ with $\operatorname{char}(F) \neq 2$. The condition $v^{2} u=(u, v, v)+v(u v)$ is an identity of $\mathcal{H}$.

Proof. Applying the associativity of $(\mathbb{H}, *)$, we have

$$
\begin{aligned}
(u, v, v)+v(u v) & =(\bar{v} * u) * v-\bar{u} *(\bar{v} * v)+\bar{v} *(\bar{u} * v) \\
& =-n(v) \bar{u}+\bar{v} *(u+\bar{u}) * v \\
& =-n(v) \bar{u}+T(u) \bar{v} * v \\
& =n(v) u .
\end{aligned}
$$

From here and by Proposition 3.3, we conclude that the identity (3) holds in $\mathcal{H}$ when $\operatorname{char}(F) \neq 2$.

We now consider $\mathrm{H}$, the standard composition algebra of type III associated to $(\mathbb{H}, *)$, over $F$. Its multiplication, denoted by juxtaposition, is given by

$$
x y:=x * \bar{y} .
$$

Theorem 3.5. Let $\mathcal{H}$ and $\mathrm{H}$ be the standard composition algebras of types $I I$ and $I I I$, respectively, associated to $(\mathbb{H}, *)$ over an algebraically closed field $F$ with $\operatorname{char}(F) \neq 2$. The algebras $\mathcal{H}$ and $\mathrm{H}$ are anti-isomorphic.

Proof. The map $h: \mathcal{H} \rightarrow \mathrm{H}$ defined by $x \mapsto \bar{x}$ is an anti-isomorphism.

Corollary 3.6. The following statements hold in $\mathrm{H}$ :

1. $e_{0}$ is its right identity;

2. there is no 2-identity;

3. all of its 3-identities are implied by $(u, u, v)=(u v) u-v u^{2}$, where $(\cdot, \cdot, \cdot)$ denotes the associator in $\mathrm{H}$;

4. $y x^{2}=n(x) y$ is an identity. 
Proof. The statements are consequences, taking into account the previous results in this section for type II, of the following fact: $I=0$ is an identity of $\mathcal{H}$ iff $h(I)=0$ is an identity of $\mathrm{H}$, where $h$ is the anti-isomorphism defined in Theorem 3.5.

\section{Some standard composition algebras asso- ciated to the octonion algebra $(\mathbb{O}, *)$}

As in the previous section, we assume that $F$ is algebraically closed. Let $(\mathbb{O}, *)$ be the octonion algebra over $F$ with basis $\left\{e_{0}, e_{1}, e_{2}, e_{3}, e_{4}, e_{5}, e_{6}, e_{7}\right\}$ and multiplication table given by $e_{i} * e_{i}=-e_{0}$ for $i \in\{1, \ldots, 7\}$, being $e_{0}$ the identity, and the below Fano plane, where the cyclic ordering of each three elements lying on the same line is shown by the arrows.

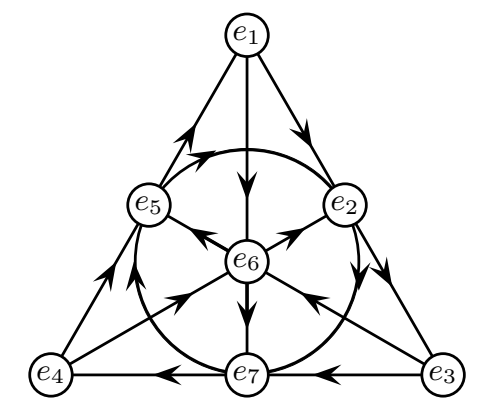

Figure 1: Fano plane for $\mathbb{D}$.

We now consider $\mathcal{O}$, the standard composition algebra of type II associated to $(\mathbb{O}, *)$, over $F$. Its multiplication, denoted by juxtaposition, is given by

$$
x y:=\bar{x} * y .
$$

Theorem 4.1. Let $\mathcal{O}$ be the standard composition algebra of type II associated to $(\mathbb{O}, *)$ over an algebraically closed field $F$ with char $(F) \neq 2$. There is no 2-identity satisfied by $\mathcal{O}$.

Proof. As $\mathcal{H}$ is a subalgebra of $\mathcal{O}$, it follows from Theorem 3.1.

Consider the subsequent table. 


\begin{tabular}{|r|r|r|r|r|r|r|r|r|}
\hline & $e_{0}$ & $e_{1}$ & $e_{2}$ & $e_{3}$ & $e_{4}$ & $e_{5}$ & $e_{6}$ & $e_{7}$ \\
\hline$e_{0}$ & $e_{0}$ & $e_{1}$ & $e_{2}$ & $e_{3}$ & $e_{4}$ & $e_{5}$ & $e_{6}$ & $e_{7}$ \\
\hline$e_{1}$ & $-e_{1}$ & $e_{0}$ & $-e_{3}$ & $e_{2}$ & $-e_{5}$ & $e_{4}$ & $-e_{7}$ & $e_{6}$ \\
\hline$e_{2}$ & $-e_{2}$ & $e_{3}$ & $e_{0}$ & $-e_{1}$ & $-e_{6}$ & $e_{7}$ & $e_{4}$ & $-e_{5}$ \\
\hline$e_{3}$ & $-e_{3}$ & $-e_{2}$ & $e_{1}$ & $e_{0}$ & $e_{7}$ & $e_{6}$ & $-e_{5}$ & $-e_{4}$ \\
\hline$e_{4}$ & $-e_{4}$ & $e_{5}$ & $e_{6}$ & $-e_{7}$ & $e_{0}$ & $-e_{1}$ & $-e_{2}$ & $e_{3}$ \\
\hline$e_{5}$ & $-e_{5}$ & $-e_{4}$ & $-e_{7}$ & $-e_{6}$ & $e_{1}$ & $e_{0}$ & $e_{3}$ & $e_{2}$ \\
\hline$e_{6}$ & $-e_{6}$ & $e_{7}$ & $-e_{4}$ & $e_{5}$ & $e_{2}$ & $-e_{3}$ & $e_{0}$ & $-e_{1}$ \\
\hline$e_{7}$ & $-e_{7}$ & $-e_{6}$ & $e_{5}$ & $e_{4}$ & $-e_{3}$ & $-e_{2}$ & $e_{1}$ & $e_{0}$ \\
\hline
\end{tabular}

Table 2: Multiplication table of $\mathcal{O}$.

Let $a=\sum_{i=0}^{7} a_{i} e_{i} \in \mathcal{O}$. Then the coordinate matrices of the left and of the right multiplication by $a$ are given, respectively, by

$$
l(a)=\left[\begin{array}{rrrrrrrr}
a_{0} & a_{1} & a_{2} & a_{3} & a_{4} & a_{5} & a_{6} & a_{7} \\
-a_{1} & a_{0} & a_{3} & -a_{2} & a_{5} & -a_{4} & a_{7} & -a_{6} \\
-a_{2} & -a_{3} & a_{0} & a_{1} & a_{6} & -a_{7} & -a_{4} & a_{5} \\
-a_{3} & a_{2} & -a_{1} & a_{0} & -a_{7} & -a_{6} & a_{5} & a_{4} \\
-a_{4} & -a_{5} & -a_{6} & a_{7} & a_{0} & a_{1} & a_{2} & -a_{3} \\
-a_{5} & a_{4} & a_{7} & a_{6} & -a_{1} & a_{0} & -a_{3} & -a_{2} \\
-a_{6} & -a_{7} & a_{4} & -a_{5} & -a_{2} & a_{3} & a_{0} & a_{1} \\
-a_{7} & a_{6} & -a_{5} & -a_{4} & a_{3} & a_{2} & -a_{1} & a_{0}
\end{array}\right]
$$

and

$$
r(a)=\left[\begin{array}{rrrrrrrr}
a_{0} & a_{1} & a_{2} & a_{3} & a_{4} & a_{5} & a_{6} & a_{7} \\
a_{1} & -a_{0} & -a_{3} & a_{2} & -a_{5} & a_{4} & -a_{7} & a_{6} \\
a_{2} & a_{3} & -a_{0} & -a_{1} & -a_{6} & a_{7} & a_{4} & -a_{5} \\
a_{3} & -a_{2} & a_{1} & -a_{0} & a_{7} & a_{6} & -a_{5} & -a_{4} \\
a_{4} & a_{5} & a_{6} & -a_{7} & -a_{0} & -a_{1} & -a_{2} & a_{3} \\
a_{5} & -a_{4} & -a_{7} & -a_{6} & a_{1} & -a_{0} & a_{3} & a_{2} \\
a_{6} & a_{7} & -a_{4} & a_{5} & a_{2} & -a_{3} & -a_{0} & -a_{1} \\
a_{7} & -a_{6} & a_{5} & a_{4} & -a_{3} & -a_{2} & a_{1} & -a_{0}
\end{array}\right] .
$$

Theorem 4.2. Let $\mathcal{O}$ be the standard composition algebra of type II associated to $(\mathbb{O}, *)$ over an algebraically closed field $F$ with $\operatorname{char}(F)=0$. The 3-identities satisfied by $\mathcal{O}$ are consequences of

$$
v^{2} u=(u, v, v)+v(u v) .
$$


Proof. We apply the random vectors method in Maple ${ }^{\mathrm{TM}}$, as in the proof of Theorem 3.2, in characteristic zero. The needed $M$ initialized to zero is a $20 \times 12$ matrix and the generated pseudo-random vectors have 8 components. By the previous notations, we write $l(w) Z$, where $w$ is given by $l(x) Y$, and $l(x)(l(y) Z)$ instead of $(x y) z$ and $x(y z)$, respectively, where $Y=\left[y_{0} \ldots y_{7}\right]^{T}$ and $Z=\left[z_{0} \ldots z_{7}\right]^{T}$. The conclusion is similar to that obtained for the degree 3 identities of $\mathcal{H}$, that is, all 3-identities of $\mathcal{O}$ are implied by $(u, v, v)=(v v) u-$ $v(u v)$, where $(\cdot, \cdot, \cdot)$ denotes the associator in the mentioned algebra.

Proposition 4.3. Let $\mathcal{O}$ be the standard composition algebra of type II associated to $(\mathbb{O}, *)$ over an algebraically closed field $F$ with $\operatorname{char}(F) \neq 2$. The condition $x^{2} y=n(x) y$ is an identity of $\mathcal{O}$.

Proof. Identical to the one of Proposition 3.3.

Theorem 4.4. Let $\mathcal{O}$ be the standard composition algebra of type II associated to $(\mathbb{O}, *)$ over an algebraically closed field $F$ with $\operatorname{char}(F) \neq 2$. The condition $v^{2} u=(u, v, v)+v(u v)$ is an identity of $\mathcal{O}$.

Proof. Although $(\mathbb{O}, *)$ is not associative, as $(\bar{x} * y) * x=\bar{x} *(y * x)$ holds in $(\mathbb{O}, *)$ and taking into account Proposition 4.3 , it is possible to prove that the identity (5) holds in $\mathcal{O}$ when $\operatorname{char}(F) \neq 2$.

We now consider 0 , the standard composition algebra of type III associated to $(\mathbb{O}, *)$, over $F$. Its multiplication, denoted by juxtaposition, is given by

$$
x y:=x * \bar{y} .
$$

As the proofs of the remaining results of this section are analogous to the ones of Theorem 3.5 and Corollary 3.6, we omit them.

Theorem 4.5. Let $\mathcal{O}$ and 0 be the standard composition algebras of types II and III, respectively, associated to $(\mathbb{O}, *)$ over an algebraically closed field $F$ with $\operatorname{char}(F) \neq 2$. The algebras $\mathcal{O}$ and 0 are anti-isomorphic.

Corollary 4.6. The following statements hold in 0 :

1. $e_{0}$ is its right identity;

2. there is no 2-identity;

3. all of its 3-identities are implied by $(u, u, v)=(u v) u-v u^{2}$, where $(\cdot, \cdot, \cdot)$ denotes the associator in 0 ;

4. $y x^{2}=n(x) y$ is an identity. 


\section{Characterization}

Notice that every standard composition algebra of type I associated to a 2-dimensional Hurwitz algebra (so, commutative and associative) satisfies the 3-identity $v^{2} u=(u, v, v)+v(u v)$ (respectively, $\left.(u, u, v)=(u v) u-v u^{2}\right)$. Hence, the former identity can not characterize all standard composition algebras of type II (respectively, III). We now prove, with $\operatorname{char}(F) \neq 2$, that the identities $x^{2} y=n(x) y$ and $y x^{2}=n(x) y$ allow us to achieve that goal.

Theorem 5.1. Let $F$ be a field with $\operatorname{char}(F) \neq 2$. Let $A$ be a composition algebra of arbitrary dimension over $F$, with multiplication denoted by juxtaposition, that satisfies

$$
x^{2} y=n(x) y .
$$

Then $A$ is a standard composition algebra of type II.

Proof. For any $x$ with $n(x) \neq 0, \frac{1}{n(x)} x^{2}$ is a left identity of $A$. In particular, there exists a left identity $e$ : $e x=x$ for any $x$. But a left identity is unique, because if $e_{1}$ and $e_{2}$ are left unities, then $\left(e_{1}-e_{2}\right) x=0$ for any $x$. As the right multiplication by any $x$ with $n(x) \neq 0$ is injective, we conclude that $e_{1}=e_{2}$. Therefore, denote by $e$ the left identity of $A$. By uniqueness, $x^{2}=n(x) e$ for any $x$ with $n(x) \neq 0$.

Let us consider the algebra obtained by scalar extension to an algebraic closure $\hat{F}: \hat{A}=A \otimes_{F} \hat{F}$.

For any $z \in A$, the subspace $S=\hat{F} e+\hat{F} z$ has finite dimension and is non-isotropic. Notice that the maps defined by $x \mapsto x^{2}$ and $x \mapsto n(x) e$, from $S$ to $S^{2}$, are polynomial functions. Since $x^{2}=n(x) e$ for any $x \in S$ with $n(x) \neq 0$, by Zariski density, $x^{2}=n(x) e$ for any $x \in S$. In particular, $z^{2}=n(z) e$. Linearizing $x^{2}=n(x) e$, we obtain $x e+e x=2 n(e, x) e$. Hence, $x e=2 n(e, x) e-x$ for any $x$ and so $r_{e}$, the right multiplication by $e$, is a linear map such that $\left(r_{e}\right)^{2}=i d$. Therefore, it is bijective.

Define a new multiplication on $A$ by $x \cdot y=r_{e}^{-1}(x) y$. Then $(A, \cdot)$ is a Hurwitz algebra (so, finite dimensional) with the same norm and identity $e$ and $x y=(x e) \cdot y$ for any $x, y \in A$.

With $x=y$ this gives $x^{2}=(x e) \cdot x$, but $x^{2}=n(x) e$, so we get $n(x) e=$ $(x e) \cdot x$ for any $x$. But $n(x) e=\bar{x} \cdot x$ (where $\bar{x}$ denotes the standard involution in the Hurwitz algebra $(A, \cdot))$. Hence $\bar{x} \cdot x=(x e) \cdot x$ for any $x$. Then $\bar{x}=x e$ for any $x$ with $n(x) \neq 0$ and, again by Zariski density on $\hat{A}$, this is valid for any $x$.

Therefore, $x y=(x e) \cdot y=\bar{x} \cdot y$ for any $x, y$, as required.

Corollary 5.2. Let $A$ be a composition algebra of arbitrary dimension over $F$, with multiplication denoted by juxtaposition, that satisfies 


$$
y x^{2}=n(x) y .
$$

Then $A$ is a standard composition algebra of type III.

Proof. If $A$ satisfies $y x^{2}=n(x) y$, then the opposite algebra $A^{o p}$ of $A$ satisfies $x^{2} y=n(x) y$. By Theorem 5.1, $A^{o p}$ is a standard composition algebra of type II. Therefore, $A$ is a standard composition algebra of type III.

\section{Acknowledgements}

The research of Beites was partially supported by Fundação para a Ciência e a Tecnologia (Portugal), project PEst-OE/MAT/UI0212/2014. Both authors were supported by Ministerio de Economía y Competitividad (Spain), project MTM2013-45588-C3-1-P.

\section{References}

[1] Beites, P. D. and Nicolás, A. P. (2014) Álgebras de composição standard de tipo II, Atas do Encontro Nacional da SPM 2014, Lisboa.

[2] Bremner, M. and Hentzel, I (2004) Identities for algebras of matrices over the octonions, J. Algebra, 277, 73-95.

[3] Chevalley, C. (1997) The algebraic theory of spinors and Clifford algebras, Springer.

[4] Elduque, A. and Pérez-Izquierdo, J. M. (1997) Composition algebras with large derivation algebras, J. Algebra, 190, 372-404.

[5] Jacobson, N. (1958) Composition algebras and their automorphisms, Rend. Circ. Mat. Palermo, 7, 55-80.

[6] Kaplansky, I. (1953) Infinite-dimensional quadratic forms admitting composition, Proc. Amer. Math. Soc., 4, 956-960.

[7] Lounesto, P. (2001) Octonions and triality, Adv. Appl. Clifford Algebras, $11,191-213$. 
[8] Okubo, S. (1982) Classification of flexible composition algebras, I and II, Hadronic J., 5, 1564-1626.

[9] Petersson, H. P. (1971) Quasi composition algebras, Abh. Math. Semin. Univ. Hambg., 35, 215-222.

[10] Stampfli-Rollier, C. (1983) 4-dimensionale Quasikompositionsalgebren, Arch. Math., 40, 516-525. 